\title{
Smoothing of rough surfaces
}

\author{
Angel Sánchez \\ Theoretical Division and Center for Nonlinear Studies, Los Alamos National Laboratory, Los Alamos, New Mexico 87545 \\ and Departamento de Matemáticas, Escuela Politécnica Superior, Universidad Carlos III, E-28911 Leganés, Madrid, Spain \\ A. R. Bishop, David Cai, and Niels Grønbech-Jensen \\ Theoretical Division and Center for Nonlinear Studies, Los Alamos National Laboratory, Los Alamos, New Mexico 87545
}

(Received 19 April 1995)

\begin{abstract}
Simulations of surface smoothing (healing) by Langevin dynamics in large systems are reported. The surface model is described by a two-dimensional discrete sine-Gordon (solid-on-solid) equation. We study how initially circular terraces decay in time for both zero and finite temperatures and we compare the results of our simulations with various analytical predictions. We then apply this knowledge to the smoothing of a rough surface obtained by heating an initially flat surface above the roughening temperature and then quenching it. We identify three regimes in terms of their time evolution, which we are able to associate with the resulting terrace morphology. The regimes consists of a short initial stage, during which small scale fluctuations disappear; an intermediate, longer time interval, when evolution can be understood in terms of terraces and their interaction; and a final situation in which almost all terraces have been suppressed. We discuss the implications of our results for modeling rough surfaces.
\end{abstract}

\section{INTRODUCTION}

The study of the morphology of growing surfaces is very important from both fundamental and applied viewpoints. ${ }^{1,2}$ Since the pioneering work of Burton, Cabrera, and Frank, ${ }^{3}$ it has been clearly established that the surface of a crystal at equilibrium is macroscopically flat at low temperature and rough above some temperature $T_{R}$, the "roughening temperature." 4 For the case of a growing surface, i.e., a nonequilibrium situation, the roughening temperature is only slightly reduced with respect to the equilibrium case (see, e.g., Ref. 5 and references therein). In view of this, the question arises as to whether an initially rough surface (i.e., in equilibrium above $T_{R}$, or growing at temperatures close to it) smooths when quenched below $T_{R}$, and if so what are the relevant time scales, and what rough features are preserved in the final structure. Posed as the study of the relaxation of a grooved surface, this problem was already discussed more than thirty years ago by Mullins, ${ }^{6}$ under the assumption that all surface properties were independent of the orientation. However, Mullins's theory fails below $T_{R}$, because the anisotropic surface energy displays cusps at particular angles. This difficulty was addressed by Martin and Perraillon ${ }^{7}$ and by Lançon and Villain, ${ }^{8}$ who showed how step structure could be taken into account. Additionally, Monte Carlo simulations showed that the atomistic structure of the surface leads to a number of new subtleties in the smoothing process (see Ref. 9 and references therein). These studies led to a good understanding of the smoothing process of a sinusoidal profile above the roughening temperature $\left(T_{R}\right)$, although the situation is less clear as regards smoothing below $T_{R}{ }^{9}$
Our purpose here is to move a step further and address the problem of the smoothing of a rough surface instead of that of an artificially grooved profile. The case of such a rough surface has not received much attention. Villain ${ }^{10}$ phrased the question as follows: For temperatures $T>T_{R}$, the surface of a crystal is rough, and the fluctuations of the height $h(r)$ diverge at long distances,

$$
C(r) \equiv\left\langle\left[h\left(\mathbf{r}^{\prime}+\mathbf{r}\right)-h\left(\mathbf{r}^{\prime}\right)\right]^{2}\right\rangle \sim C(T) \ln r,
$$

where $C(T)$ is a temperature-dependent constant, $\mathbf{r}$ and $\mathbf{r}^{\prime}$ are two-dimensional (2D) vectors giving the position at which height is measured, and $r \equiv|\mathbf{r}|$. If now the crystal is cooled down, from $T>T_{R}$ to $T \ll T_{R}$, it is still rough. How does the roughness decrease with time $t$ ? From the theoretical analysis in Ref. 10, there were two main conclusions: On the one hand, smaller terraces disappear first, and after a time $t$ the minimum terrace size $R_{\min }^{3} \sim t \exp \left(-E_{0} / T\right) / T$ (disregarding a few small terraces due to shrinkage of large ones); on the other hand, for an infinite surface $C(r)$ is still given by the same expression as above, and only at short distances is the height difference much smaller. The prediction about $R_{\min }$ was in contradiction with the results in Ref. $6\left(R_{\min }^{4} \sim t\right)$ and in Ref. $7\left(R_{\min }^{2} \sim t\right)$, and therefore, as noted by Villain in Ref. 10, there are subtleties in the theoretical treatment of the problem.

Motivated by this unclear situation of sinusoidal profile smoothing below $T_{R},{ }^{9}$ and by the lack of any experimental or numerical result on smoothing of fully rough surfaces, in this work we undertook the study of this problem by means of Langevin dynamics simulations of a $2 \mathrm{D}$ discrete sine-Gordon ( solid-on-solid) model. We report on our results as follows. In Sec. II we present our 
model and simulation procedure. In Sec. III we study the decay of a single circular terrace, i.e., precisely the situation studied analytically in Ref. 10. We present results for both zero and finite temperatures and compare them to the theoretical predictions of Ref. 10. We describe several characteristics of the decay and show how measurable quantities like $C(r)$ or roughness can be used to follow the surface evolution. In Sec. IV we turn to rough surface decay, and show how the results on single terraces can be used to understand this more complex situation. We identify the three main stages of the decay, namely decay of small fluctuations, rounding and fusion of terraces, and decay of terraces, again both at zero and finite temperature. Finally, in Sec. V, we summarize our conclusions, and discuss smoothing of rough surfaces from a general viewpoint connecting our simulations and Villain's conjectures. We finish with a discussion of possible consequences of our work.

\section{MODEL AND SIMULATION PROCEDURE}

The model we study to describe surface growth phenomena is given by the following $2 \mathrm{D}$ sine-Gordon-like potential introduced in Ref. 11:

$$
V=\frac{1}{2} \sum_{\langle i, j\rangle}\left(\phi_{i}-\phi_{j}\right)^{2}-\sum_{i} \cos \phi_{i},
$$

with $\phi_{i}$ being a continuous variable on a discrete square lattice. The first term describes the interaction energy between a column at site $i$ and its nearest neighbors, representing surface tension, and the second term favors $\phi_{i}$ to be $2 \pi n$ (so we can make the identification $\phi=2 \pi h$, $h$ being the height) and is directly related to periodic pinning effects of the lattice. This model is a faithful representation of the discrete Gaussian solid-on-solid model of surface growth. The equilibrium roughening temperature was determined in Refs. 11 and 12 and found to be in very good agreement with renormalization group predictions $^{13}$ (see also Ref. 4 and references therein). More recently, the roughening transition has also been studied in the nonequilibrium case, and it was found that the equilibrium transition splits into two rapid crossovers or transitions separating very different growth regimes. ${ }^{5}$ Furthermore, recent experiments ${ }^{14}$ on growth of GaAs by metalorganic vapor phase epitaxy (MOVPE) exhibit morphologies very similar to those obtained with model (2), as reported in Ref. 11. These data suggest that this model indeed captures much of the essential physics of surface growth phenomena.

We study model (2) by means of Langevin dynamics simulations, integrating the overdamped Langevin equations of motion for $\phi$ with interaction given by Eq. (2), which read

$$
\dot{\phi}_{i}=\sum_{\langle i, j\rangle}\left(\phi_{j}-\phi_{i}\right)+\sin \phi_{i}+\xi_{i}(t),
$$

where the overdot means time derivative and $\xi_{i}(t)$ is a Gaussian white noise of mean and variance

$$
\left\langle\xi_{i}(t)\right\rangle=0,\left\langle\xi_{i}(t) \xi_{j}\left(t^{\prime}\right)\right\rangle=2 \alpha T \delta_{i j} \delta\left(t-t^{\prime}\right)
$$

in units such that $k_{B}=1$. Our numerical procedure is a fast ${ }^{15}$ implementation of a stochastic RungeKutta method, ${ }^{16}$ which allows the study of lattices up to $1024 \times 1024$ sites on workstations, without resorting to a massively parallel computer as in Ref. 11. These computational advances make Langevin dynamics an attractive method for nonequilibrium statistical mechanics problems such as surface growth or Josephson junction arrays. $^{17}$

\section{SMOOTHING OF SINGLE TERRACES}

We begin our study of the decay of rough surfaces from the same starting point as in Ref. 10, where it was assumed that the surface consisted of broad terraces parallel to the equilibrium surface direction and separated by steps. The analysis of the decay of these terraces proceeded under the constraint that they remain circular. Following the same approach, we have carried out a number of simulations with initial condition given by a circular terrace concentric with our simulation lattice, i.e., if the system size has $N=L \times L$ sites,

$$
\phi_{i}(0)=\left\{\begin{array}{lr}
2 \pi & \text { if }\left|\mathbf{r}_{i}-\mathbf{r}_{N / 2}\right| \leq R \\
0 & \text { otherwise }
\end{array}\right.
$$

It is clear that the constraint $R<L / 2$ has to be kept in mind. We have performed Langevin dynamics simulations of the evolution of these initial data under Eqs. (3) for both zero and finite temperature, and we collect our results for these two cases below. Before beginning the report, we stress that this initial condition is rather artificial, and that the purpose of such a study is not to compare it directly to actual growth phenomena. Rather, we intend to verify Villain's results ${ }^{10}$ as well as to learn from this simpler smoothing process how to interpret more complex ones later.

\section{A. Zero temperature}

At zero temperature the evolution of the terrace is purely deterministic. In addition, there are no fluctuations which can mask the evolution of the terrace, and therefore results related to terrace extinction times as well as other quantities of interest are easily obtained from the raw simulation data. The behavior of the terrace is as expected: its area steadily diminishes until it eventually vanishes, leaving a completely flat surface. We have monitored this process by means of several measures: global quantities, such as mean height $\left\langle\phi_{i}\right\rangle$ or mean roughness $w^{2} \equiv\left\langle\left(\phi_{i}^{2}-\left\langle\phi_{i}\right\rangle^{2}\right)\right\rangle$, and functions like $C(r)$, already introduced, or $w(r)$, defined as the mean roughness computed over regions of size $r$. All these data were of course complemented by direct analysis of surface plots, which allowed us to relate the evolution of those quantities to the evolution of the surface.

We begin by discussing global data, an example of which is shown in Fig. 1: All the quantities in this plot are seen to behave linearly with time, or almost linearly 

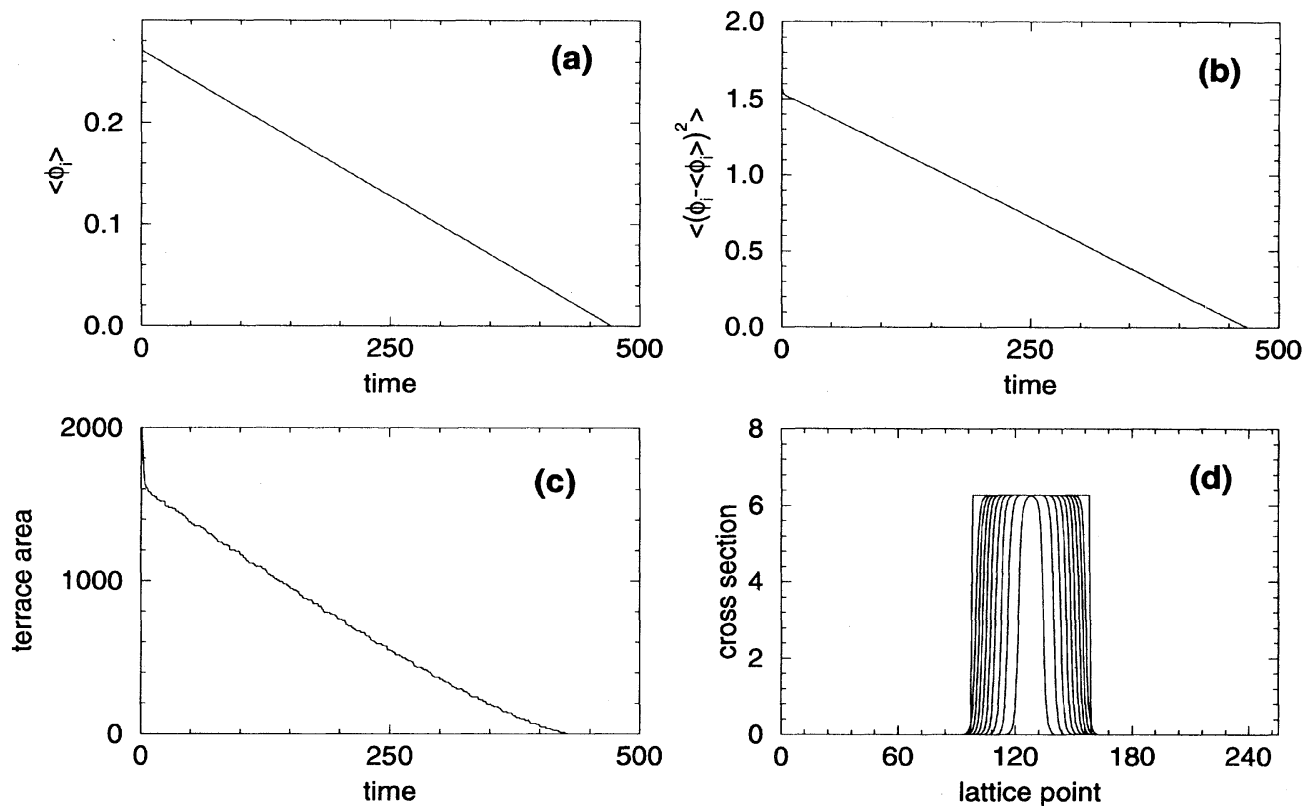

FIG. 1. Decay of a single terrace at zero temperature. Initial radius is $R_{0}=30$ and the lattice consists of $256 \times 256$ sites. Shown are the mean height (a), the mean roughness (b), the mean area of the terrace as given by the surface area above $2 \pi$ (c), and the evolution of a cross section (through the middle of the lattice) as given by snapshots taken every 50 time units (d). in the case of the terrace area. This last quantity is defined as the number of lattice points whose height is greater than or equal to $2 \pi$. We stress that in the particular case of Fig. 1 the base terrace (i.e., the whole lattice) had $256 \times 256$ sites, but we have verified that larger sizes, as well as larger upper terraces, give the same results. The plot of the cross section of the terrace in Fig. 1(d) confirms what one expects. The terrace evolves smoothly, until it vanishes, this last stage of the process being somewhat faster. The circular shape of the terrace is preserved throughout. In view of all these indicators, the time at which the terrace is adsorbed into the substrate is easily determined; in the case of the example in Fig. 1, it is around 470 in our units. Note that the terrace area vanishes prior to the time at which mean height and roughness do, this being a trivial consequence of the definition of the area as the number of points with $\phi_{i} \leq 2 \pi$ : points with $0<\phi_{i}<2 \pi$ still contribute to mean height and roughness but contribute zero to the area. This introduces some freedom in assigning the terrace extinction time: unless explicitly stated otherwise, when giving these times we will be referring to the vanishing of the area. We thus are in a position to make a first attempt at verifying Villain's result ${ }^{10}$ about $R_{\text {min }}$, by simulating the decay of terraces of $2 \mathrm{D}$ different sizes. The results are shown in Fig. 2. They show clearly that at zero temperature $R_{\min } \sim t^{1 / 2}$ (the exponent is $0.495 \pm 0.005$ as obtained from the fit), instead of the predicted exponent, 1/3. This is a sensible result in view of the structure of the equations of our model, which in the absence of temperature (noise) are just a set of diffusion-type equations. In addition such behavior is consistent with the use of this model as an evaporationcondensation mechanism for growth. ${ }^{9}$

The data for zero temperature evolution are also useful to relate the changes in the correlation function $C(r)$ or in the roughness $w(r)$ to the terrace decay. This is important because it is one of the best ways to charac- terize the structure of the surface, and the hope is that it will provide insight when dealing with fully rough initial data. Figure 3 shows a sequence of correlation functions $C(r)$ obtained at different times, which turns out to be a good indicator of the terrace radius. This is clearly seen from the beginning of the constant plateau, which occurs precisely at the diameter of the terrace, being nontrivial for smaller distances. To understand this conclusion, it is important to notice that $C(r)$ is the height-difference correlation function, so that sites separated by more than the diameter of the terrace must have the same value of this function everywhere. Figure 4 shows how the roughness behaves while the terrace is shrinking. In this case, it can be seen from its definition (taking into account that the regions to compute the value of $w^{2}$ are chosen to be squares of side $r$ ) that its peak also coincides with the terrace size, and therefore the information this func-

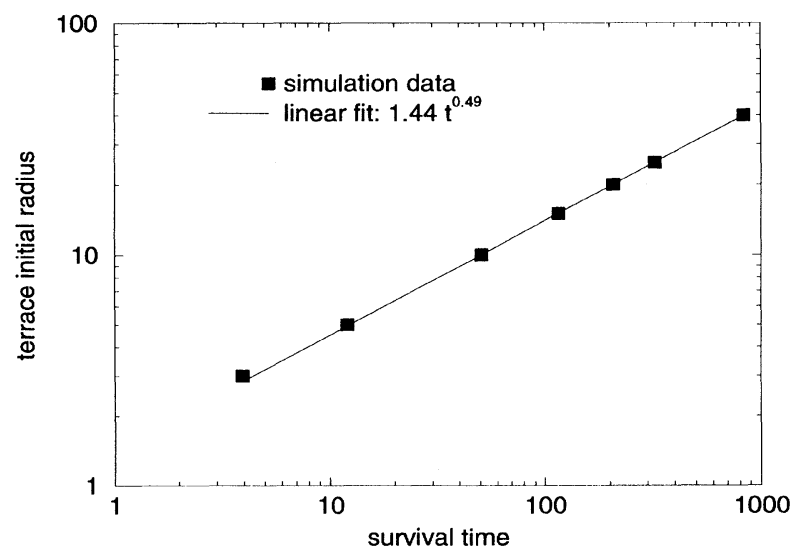

FIG. 2. Log-log plot of the radius of the initial terrace vs its survival time, i.e., the elapsed time until the surface has height $\phi_{i}<2 \pi$ everywhere. Fitted data are a least-squares straight line. 


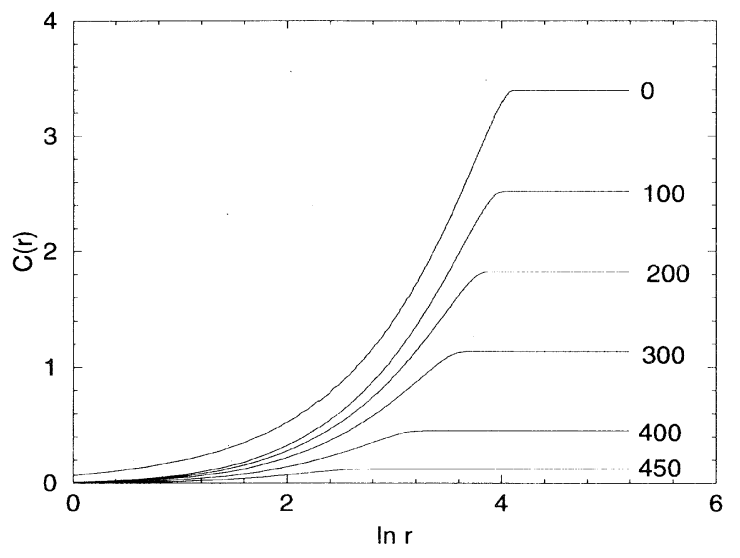

FIG. 3. Log-normal plot of $C(r)$ at different times during the simulation in Fig. 1.

tion provides is another confirmation of the picture we have described.

\section{B. Finite temperature}

Having considered how terraces decay at zero temperature, we can move a step forward and consider the smoothing problem at small, but finite temperatures. As in the previous subsection, we begin by studying how global quantities evolve; an example of the behavior of these data is shown in Fig. 5 for $T=5$. The choice of the temperature value is rather arbitrary, and in principle the only constraint for Villain's theory ${ }^{10}$ to be applicable is $T \ll T_{R}$ with $T_{R} \sim 27$ in our units. ${ }^{5}$ Note that the plots in Fig. 5 correspond to the evolution of one specific terrace and that for the moment we postpone the discussion of mean terrace behavior. At first sight, the conclusion that can be drawn from this simulation is that the results for $T=5$ are basically the same as those obtained at $T=0$, the main difference being a more noisy appearance which evidently comes from thermal fluctuations. Probing deeper into the details of the

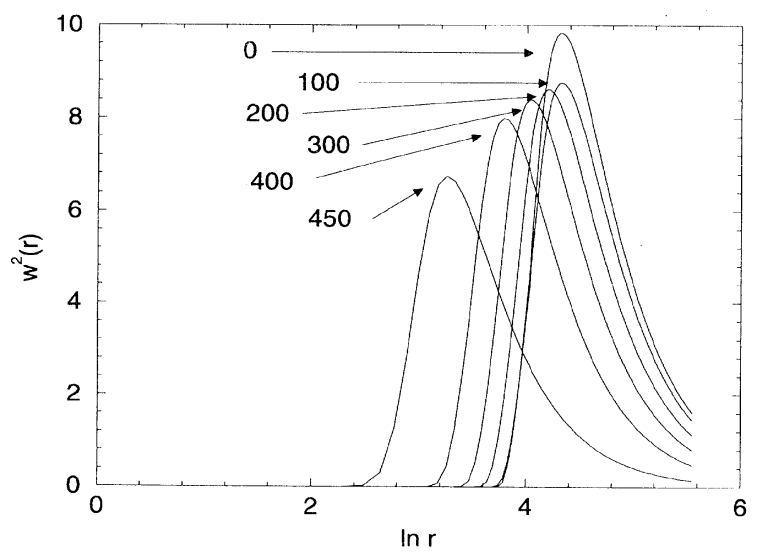

FIG. 4. Log-normal plot of $w^{2}(r)$ at different times during the simulation in Fig. 1. plots, another feature arises due to temperature. Thus, in Figs. 5(a) and 5(c), it can be observed that the time for the terrace to disappear, about $t=600$, is larger than the survival time at $T=0$ [cf. Figs. 1(a) and 1(c)]; however, after the terrace disappears the height fluctuates around $\phi=0$ as in the previous case. This does not occur with roughness: After the terrace dies out, roughness asymptotes to a nonzero constant value. The shape of the terrace, although not exactly circular, remains approximately circular throughout the evolution and the terrace itself is always a separate entity, distinguishable from the background [see Fig. 5(d)]. As in the case of zero temperature, both the correlation function $C(r)$ (Fig. 6) and the roughness $w^{2}(r)$ (Fig. 7) confirm the evolution and the determination of the survival time obtained from global quantities. Note that there is a jump in $C(r)$ from its shape at $t=0$ to those at $t>0$. This jump is a consequence of the time elapsed between the first and the second snapshot, during which the $C(r)$ profile corresponding to $T=5$ and due to thermally induced correlations is established. ${ }^{5}$

In the preceding paragraph, we have argued that at finite temperature, specifically at $T=5$, the evolution of a single terrace is rather similar to what takes place at $T=0$. However, this assertion is somewhat simplistic and deserves further consideration. In the first place, upon increasing temperature, the evolution of the terrace is more and more blurred by the thermal fluctuations of its surroundings, and to pinpoint a survival time becomes subjective. An example is given in Fig. 8, where we plot the mean height [Fig. $8(\mathrm{a})]$ and the terrace area [Fig. 8 (b)] for $T=10$ and $T=15$. Whereas for the first value one can still conclude that the terrace disappears around $t=400$, in the second case it is difficult to learn anything at all (as well as from the other indicators we have used). Of course, the value of the temperature above which it is impossible to follow the evolution of the terrace depends in part on its initial size: For larger terraces the initial values of mean height and area are larger and in the beginning they decrease more or less linearly. This is consistent with the coarse-graining picture of different scales involved. However, eventually these quantities fall below their corresponding thermal values and one loses track of the terrace evolution. Due to this problem, in our simulations we have not been able to obtain accurate values for terrace decay times above $T=10$, and other indicators will be needed for smoothing so close to $T_{R}$.

Another question we have considered is the mean behavior of terraces of a given size. From our simulations, for the same initial condition different evolutions yield large discrepancies in the survival time. We illustrate this point in Fig. 9, where we show an average over ten evolutions of the same initial condition, namely, a terrace with radius $R=25$. In this plot, the error bars are obtained as the mean square root of the dispersion and, as can be seen, they span a wide interval around the mean height behavior. Once again, other quantities depend on time in much the same way as this example. From a linear fit to the mean height evolution, it is found that the survival time is $t \sim 380$, but if fluctuations around the mean are included then the result should be given 

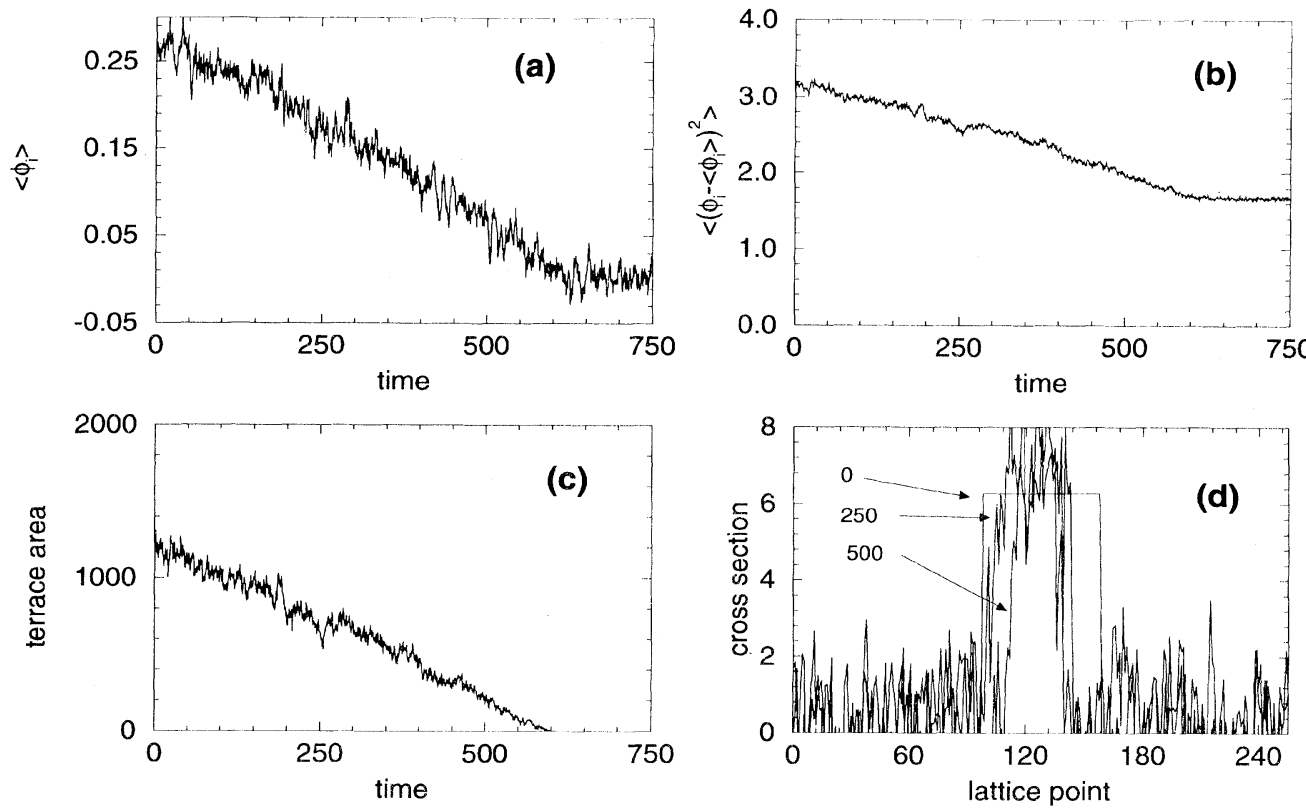

FIG. 5. As in Fig. 1 but for temperature $T=5$. In plot (d), snapshots are taken every 250 units to clarify the graph.

as $t=380 \pm 50$, which introduces an uncertainty above $10 \%$. A similar value can be estimated from terrace area analyses. Larger ensembles would be necessary to clarify or improve this bound, but those would consume much CPU time, and we believe that this band will not be much narrowed, the dispersion of different realizations being a natural consequence of thermal fluctuations. In any event, if one relies on the survival time so extrapolated from this kind of ensemble averages, the results are not very conclusive, as can be understood from Fig. 10, where we show the same kind of plot as Fig. 4, namely, terrace initial radius vs survival time but for our finite temperature data. We see that the simulations for $T=5$ still yield an approximate straight line in our log-log plot, giving an exponent of about $0.44 \pm 0.01$. This is clearly smaller than the $1 / 2$ exponent found at zero temperature, a fact which might indicate that finite temperature introduces corrections to the exponent. Nevertheless, we

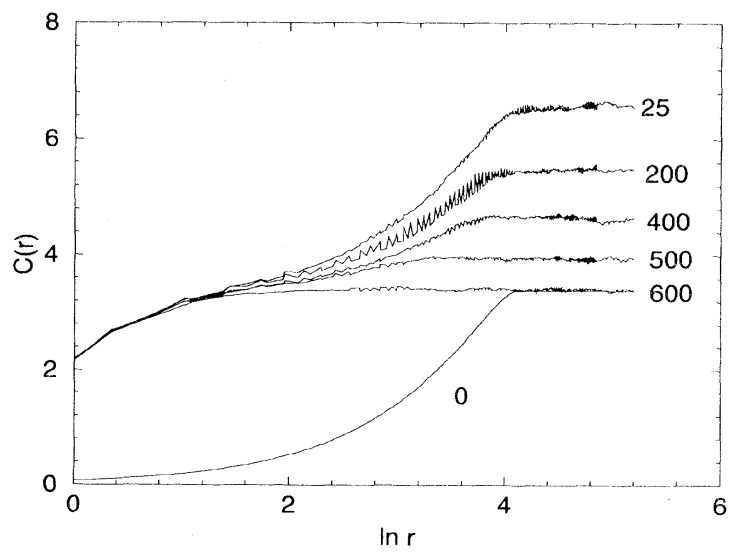

FIG. 6. Log-normal plot of $C(r)$ at different times during the simulation at $T=5$ in Fig. 5 . also show a few points obtained at $T=10$. We observe that in this case the dispersion is even larger, as should be expected, and as a consequence our values are less reliable. These are further compromised by the already mentioned difficulties in pinpointing the precise time at which the terrace dies out. Therefore, the exponent obtained from this fit, a bit larger than $1 / 2$, is also not very reliable. Our conclusion for those and larger values of the temperature is that significantly larger ensembles are necessary.

We conclude this section by introducing an additional measure, namely the fractal dimension of the terrace. We obtain this quantity as follows. As in the case of the area, we regard points at which the height of the surface is above $2 \pi$ as belonging to the terrace. With that definition, we then compute the fractal dimension of the terrace surface according to the standard box-counting procedure. ${ }^{18}$ We have used the smooth evolution occur-

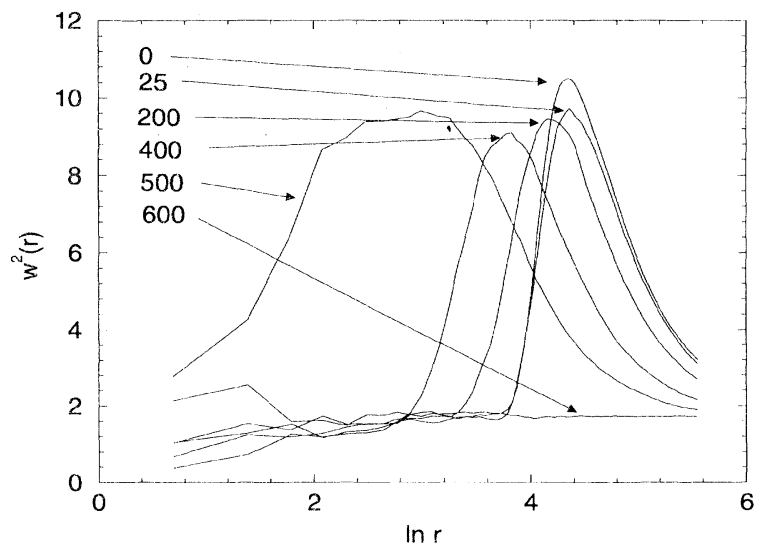

FIG. 7. Log-normal plot of $w^{2}(r)$ at different times during the simulation at $T=5$ in Fig. 5. 

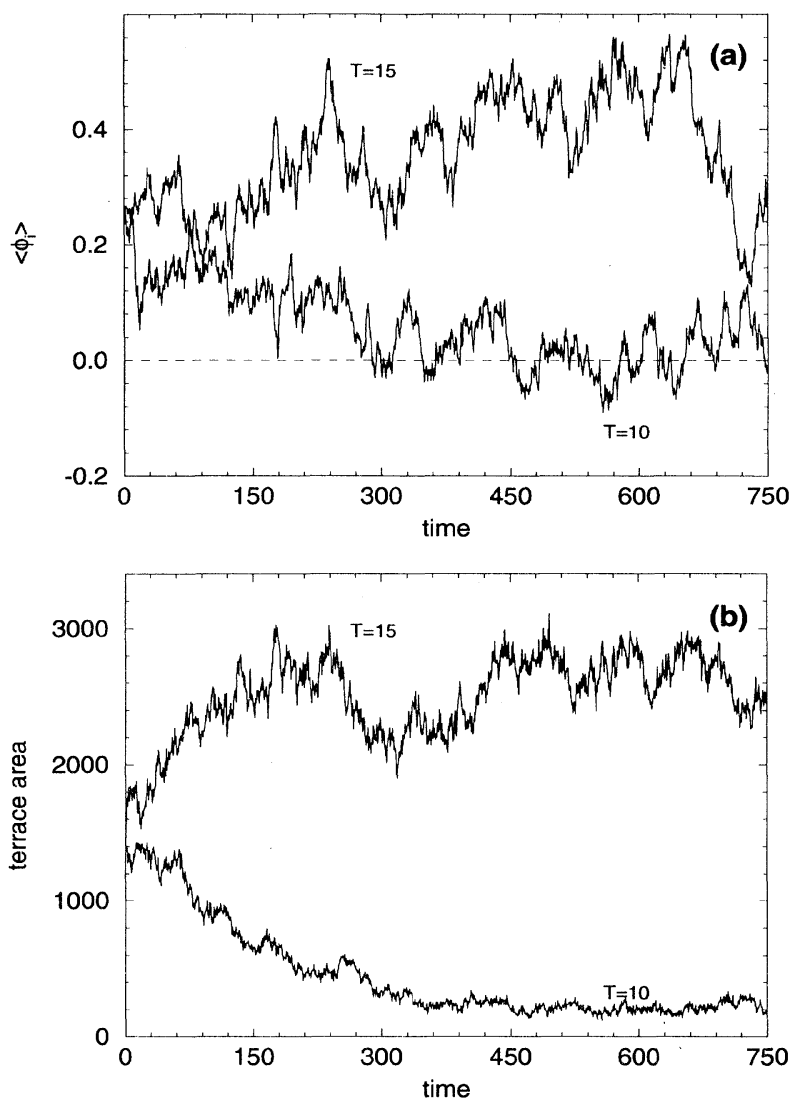

FIG. 8. Decay of a single terrace at $T=10$ and $T=15$ as seen through the evolution of its mean height (a) and its area (b). Initial radius is $R_{0}=30$ and the lattice consists of $256 \times 256$ sites.

ring at zero temperature, where the terrace is always flat at its top, to check our computation procedure with satisfactory results. An example of the results for large terraces at finite temperature is given in Fig. 11. Figure 11(a) shows the evolution in time of the box-counting dimension, leading to the conclusion that it consists of a

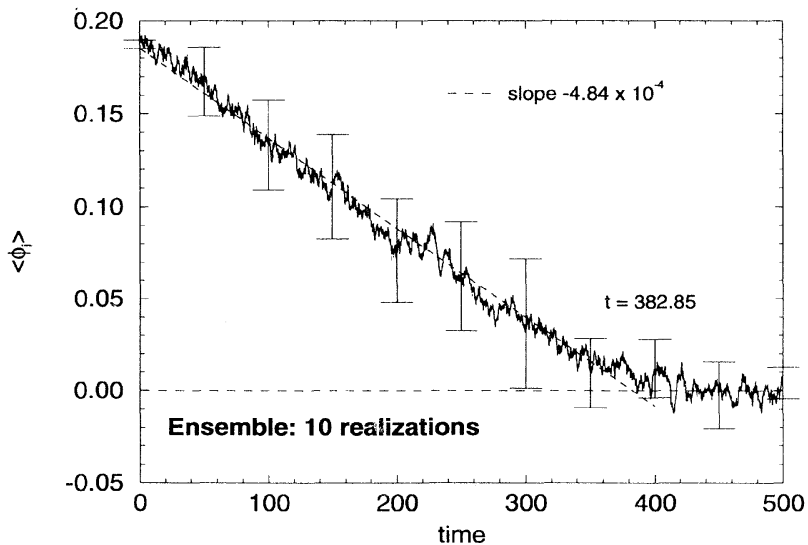

FIG. 9. Average decay of single terraces at $T=5$. Initial radius is $R_{0}=25$ and the lattice consists of $256 \times 256$ sites. The mean height is shown for an ensemble of ten realizations.

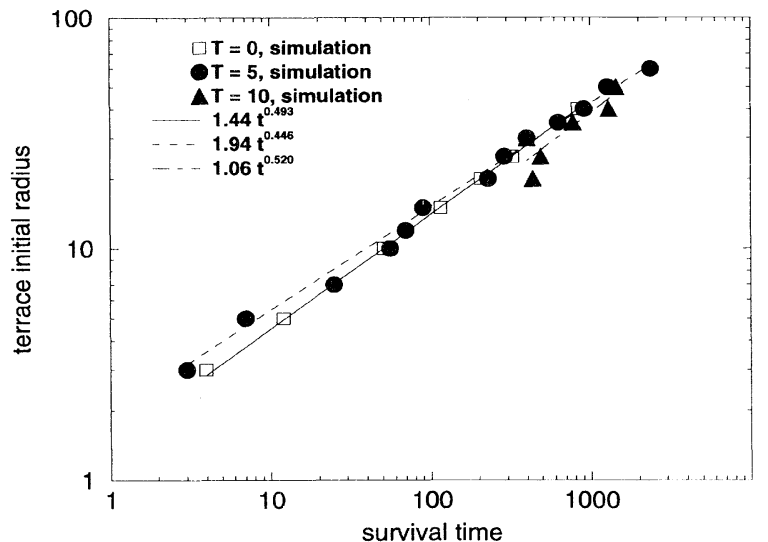

FIG. 10. Log-log plot of the radius of the initial terrace vs its extinction time at $T=5$ and $T=10$. Fitted data are a least-squares straight line. The fit of data for $T=10$ is not very good. Data for $T=0$ in Fig. 2 are included again for comparison.
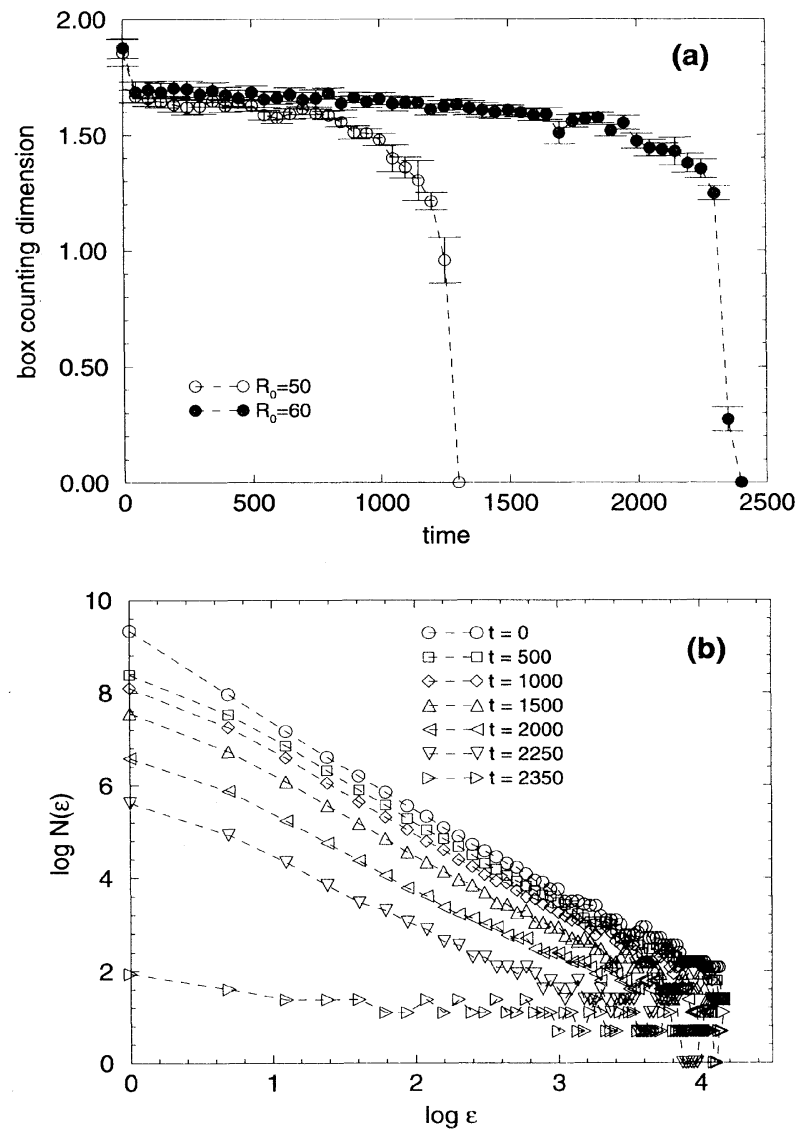

FIG. 11. (a) Box-counting dimension of the terrace, computed taking into account the points which are above $2 \pi$, vs time. Initial radii are $R_{0}=50$ and $R_{0}=60$, the lattice consists of $256 \times 256$ sites, and the temperature is $T=5$. (b) Box-counting scalings from which the dimension is computed for the case $R_{0}=50$. Note that the power-law regime necessary for the dimension to be meaningful is very well verified. 
rapid decay phase, followed by a plateau which comprises most of the terrace lifetime, and a final smooth but fast decay. The first and third stages have a duration which is mostly independent of the initial terrace size, this being the reason why we show data for terraces twice as large as the ones considered so far: the early transient and the final phase of the evolution span the lifetime of smaller terraces, making it impossible to notice the intermediate plateau. This plateau has a deep significance: it reflects the fact that during its shrinking the terrace remains a separate object with the same statistical structure. This is confirmed by Fig. 11(b), which proves the existence of a sufficiently large scaling regime in the box-counting dimension computation to consider the terrace surface a fractal in its own right. We do not show results for larger temperatures because in that case, as discussed above, there are many points of the lattice with $\phi_{i}>2 \pi$ which do not belong to the terrace, therefore preventing the possibility of identifying it and computing its fractal dimension.

\section{SMOOTHING OF ROUGH SURFACES}

We now turn to the study of how actual rough surfaces behave when quenched well below the roughening temperature. For these simulations, initial conditions are produced by starting from a perfectly flat $\left(\phi_{i}=0\right.$ for all sites) lattice and letting the system evolve at $T>T_{R} \sim 27$ long enough for roughness to be established. It is clear that these kinds of initial data are much closer to real growth processes than the terraces we have been considering so far, and consequently understanding how these rough surfaces evolve should be our main objective. As we will summarize below, it turns out that most of the evolution can be interpreted in terms of what we have learned in the single terrace studies. We will again present our results in separate subsections for zero and finite temperatures.

\section{A. Zero temperature}

We begin the discussion of the results for $T=0$ by presenting the behavior of the same quantities we used to describe the evolution of single terraces. This is contained in Fig. 12, where the main differences between terrace and rough surface decay can be readily appreciated. The mean height is a nonmonotonic function of time [see Fig. 12(a)]: Upon quenching (at $t=100$ the temperature is set to $T=0$ ), the mean height increases, reaches a maximum, and then decreases, crossing zero and behaving piecewise linearly afterwards. Interestingly, there are two intervals during which the mean height remains constant. We will return to this point once we have understood the structure of the surface. The next measure, the mean roughness, is seen from Fig. 12(b) to behave monotonically, steadily decreasing in a way that is reasonably approximated as a stretched exponential as indicated in the plot. However, the last part of the evolution may as well be linear, and at this point we cannot reach a definite conclusion. The other global quantity analyzed is the terrace area, shown in Fig. 12(c), which was defined as the number of points in the lattice having height $\phi_{i} \geq 2 \pi$. In the problem we deal with now, as the initial condition does not contain a single terrace, we cannot associate this quantity directly with a terrace area, although we will still retain that name for it. However, it is still useful, as the information it provides complements that of the mean height. Thus both quantities behave similarly initially, increasing and simultaneously reaching a maximum, but afterwards the terrace area monotonically decreases and its evolution seems to be linear. Finally, the last plot in Fig. 12 again shows a cross section of the
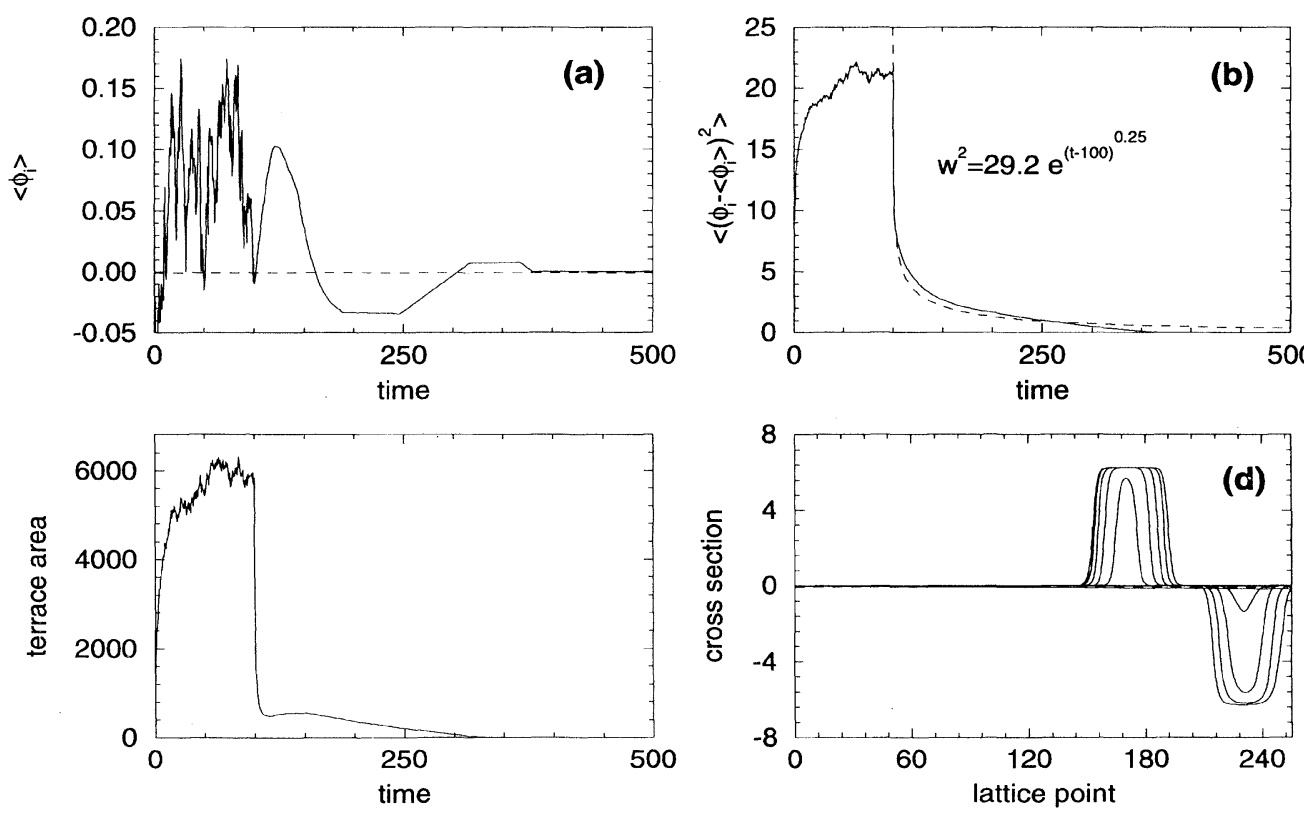

FIG. 12. Decay of a rough surface at zero temperature. The initial data are built by annealing a flat surface at $T=30$ during an interval of 100 time units, and the lattice consists of $256 \times 256$ sites. Shown are the mean height (a), the mean roughness (b), the mean area of the terrace as given by the surface area above $2 \pi$ (c), and the evolution of a cross section (through column 15) as given by snapshots taken every 50 time units (d). 
surface, through a lattice column that is seen to intersect two terraces. This figure suggests that the structure of the smoothing surface consists of terraces and motivates us to take a closer look at its profile.

The evolution of the surface as it smooths is depicted via contour plots in Fig. 13. Heights are coded as grayscale tones, points corresponding to white (black) zones being the ones with largest positive (negative) heights [the convention can always be checked by comparing these contour plots with the cross sections in Fig. 12(d) taken through column 15]. The surface situation exactly before the quench from $T=30$ to $T=0$ is plotted in Fig. 13(a). Thermal fluctuations give an approximately homogeneous aspect to the surface, although

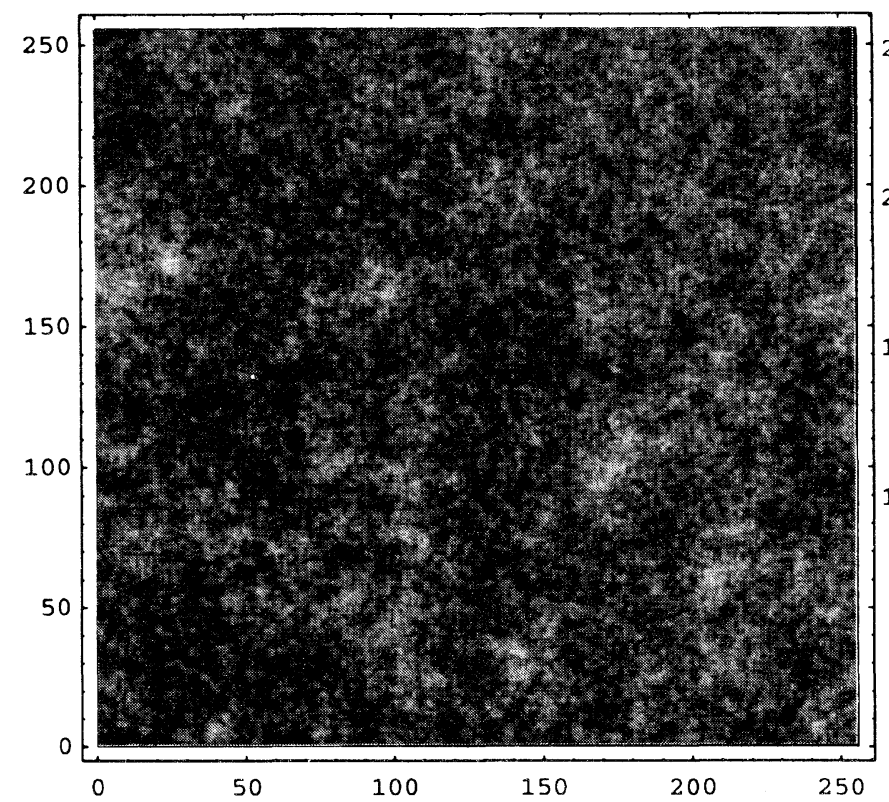

(a)

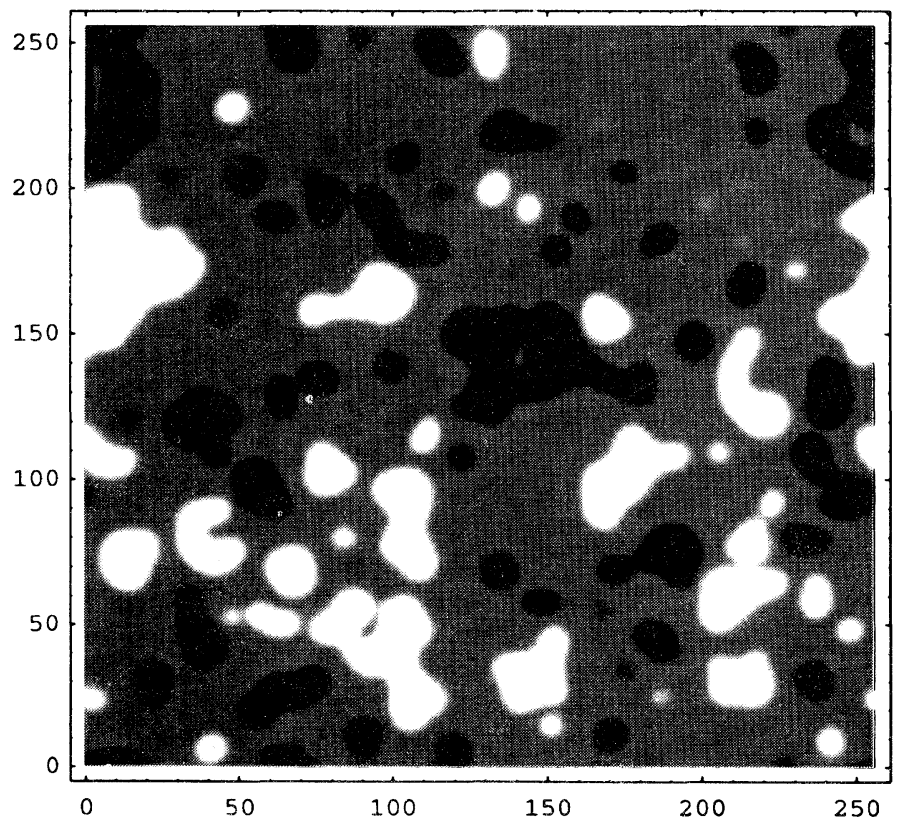

(c)

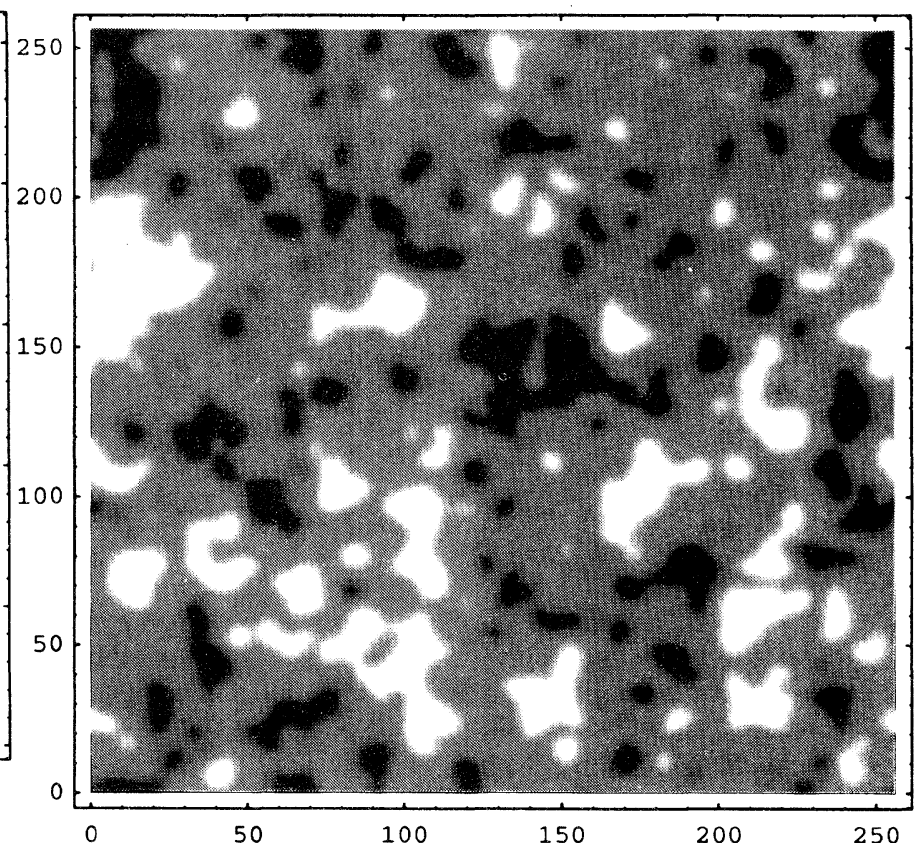

(b)

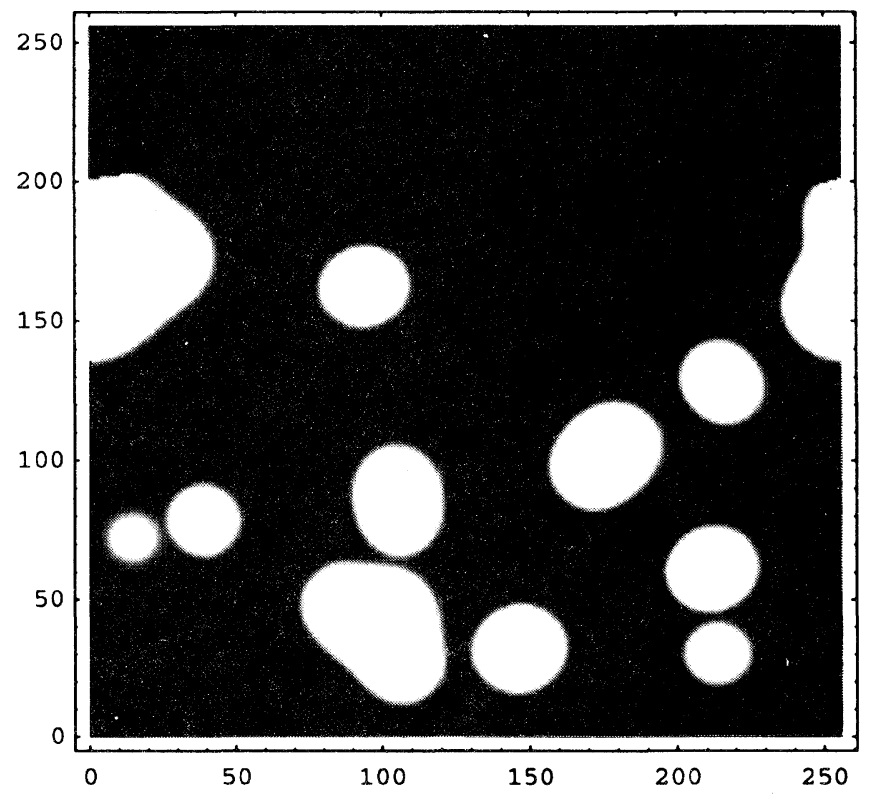

(d)

FIG. 13. Contour plots of the shape of the surface at times immediately before quenching (a), $t=5$ after the quench (b), $t=10$ after the quench (c), $t=50$ after the quench (d), and $t=100$ after the quench (e). The grayscale codes height such that lighter areas correspond to points with larger height. Axes simply indicate the coordinates of the lattice node, and periodic boundary conditions must be kept in mind. The evolution is the same as in Fig. 12. 


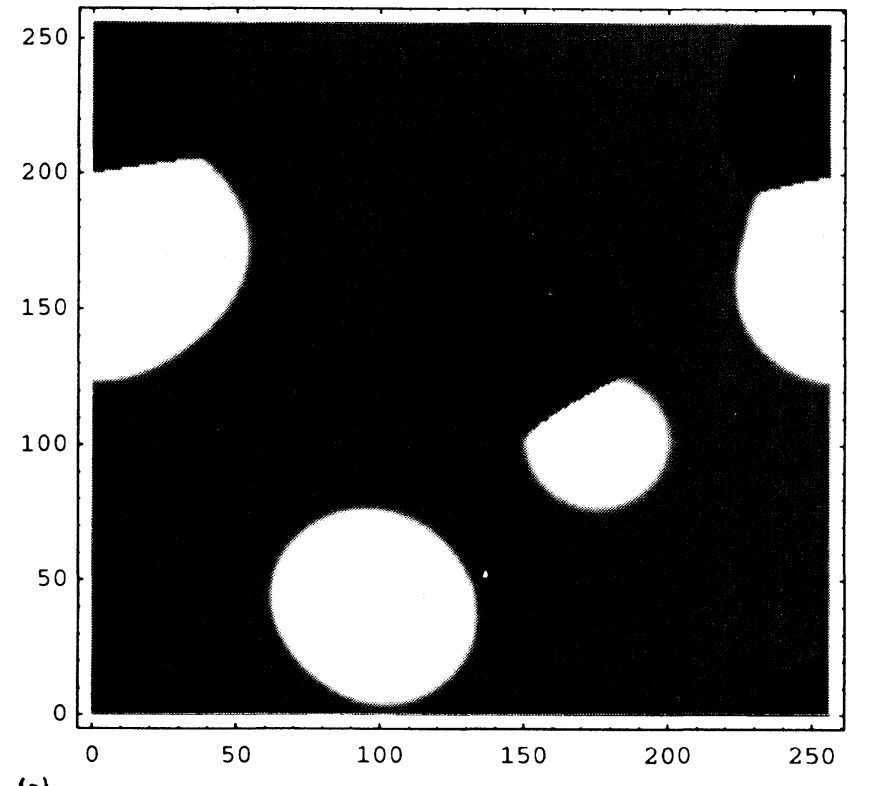

(e)

FIG. 13 (Continued).

there are higher (in mean) zones [e.g., around points $(30,180)$ or $(100,40)]$ as well as deeper (in mean) zones [e.g., around points $(140,140)$ and $(10,220)]$. Of course, as we are above the roughening temperature, larger lattices would show points with even higher heights: the maximum of this simulation is around $\left|\phi_{i}\right| \approx 10$. Those higher or deeper regions serve as nucleation centers as soon as the surface is quenched, as can be seen from Fig. 13(b). This contour plot implies that there is a first, rapid stage of the evolution during which all small scale fluctuations die away, in accordance with the fact that in 5 time units the surface has become clearly inhomogeneous, consisting of terraces (or wells) on an otherwise flat surface. Such a stage is seen also in the global quantities (Fig. 12) in their initial abrupt decay. Thus, 5 time units after the quench the surface structure can already be described in terms of terraces, which have formed above or below the initial condition with approximately equal probability, leading to a mean height close to zero.

Once the terrace structure has been built up from the initial condition, a second stage of the evolution begins. At this moment, the largest terraces, which had ramified shapes as a consequence of their origin in the quenching of fluctuations, start rounding out, engulfing lower zones which were embedded within them [cf. Fig. 13(c), the well close to the lattice center, or the terrace centered around $(120,40)]$. Also, neighboring terraces interact, and in some cases the larger one absorbs the smaller one [see Fig. 13(c), the terraces near $(220,70)$ ]. These kinds of processes are the ones leading to an increase of the mean height and terrace area (see Fig. 12). During such rearranging, the smallest terraces are already disappearing, as predicted by Villain's power-law relationship ${ }^{10}$ between terrace radii and survival times: An example appears in Fig. 13(b), which shows two small terraces around $(235,195)$ that are absent already in Fig. 13(c).
This second stage lasts some 50 time units, in fact until the mean height and area reach their maxima; the status of the surface at that moment is shown in Fig. 13(d). The surface consists now of large terraces, with only a few arising from shrinking steps of radius $R<R_{\min } \approx 20$. This is just the picture proposed in Ref. 10 . We are now in the third and final stage, governed by the superposition of the decay of the different terraces, which no longer interact. At $t=100$ after the quench, only three terraces and two wells remain, with approximately the same size [see Fig. 13(e)].

It is now easy to interpret the behavior of the mean height [Fig. 12(a)]: during the first plateau, all terraces are decreasing, but they are still rather large; hence, they decay at a very similar rate, and thus terraces and wells compensate each other's contribution to the mean height, which remains practically constant. Finally, one of the wells is small enough, its shrinking becomes faster, and as a consequence the mean height increases, becoming positive although closer to zero. At this point only the two terraces shown in Fig. 12(d) survive. Similar reasoning accounts for the following plateau and the final decay to zero, as well as for the area (which only includes terraces with positive height, this being the reason for its monotonic decay). We can also now provide an explanation for the stretched exponential shape of the roughness [see Fig. 12(b)]. This quantity has to decrease as the surface becomes more and more uniform, uniform meaning not only flat but with the terraces being similar or approximately of the same size. This decreasing takes place of course as, first, small fluctuations are quenched, followed by the slower decay of larger fluctuations (not large enough to nucleate a terrace) and the first, smallest terraces. Clearly, this is a many scale process, as there are shrinking terraces of very many sizes (more sizes if larger systems are considered). The stretched exponential behavior should be understood as arising from this many scale (glassylike) process.

It remains to be discussed how the above process and the interpretation we have provided in terms of three stages are reflected in the height-height correlation function and in the roughness. These two functions are shown in Figs. 14 and 15, and offer basically the same information as in the case of single terraces. Focusing on the correlation function $C(r)$ depicted in Fig. 14, we notice the rapid suppression of small fluctuations in the fast, global drop of the values of this function as a whole (compare the two uppermost curves in the plot). The second stage is somewhat more difficult to observe, although it does appear in that the point at which the curve reaches the asymptotic plateau moves to higher $r$ values. Recalling our discussion of the preceding section (that this point indicates the size of the terrace), it is readily noticed that this value of $r$ is now associated with the size of the largest terraces present in the surface. Also, the disappearance of the smallest terraces is seen through $C(r)$ in the small $r$ limit. The flattening of the curve in this region comes from the fact that there are no features of that scale, i.e., no small radius terraces. Finally, the remainder of the evolution, which is the third stage understandable in terms of single terrace decay, coincides 


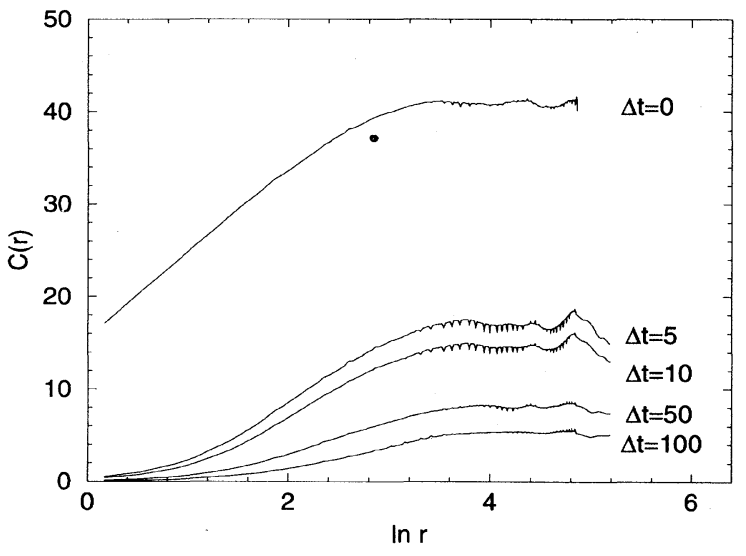

FIG. 14. Log-normal plot of $C(r)$ at the same times given in Fig. 13.

with what we argued in the previous section. As for the roughness behavior, the plot of $w^{2}(r)$ in Fig. 15 is amenable to the same interpretation, even if in this case the data for the surface before the quenching do not have a definite shape. There is again a rapid drop, after which curves start taking a shape close to that of single terraces. This is somewhat blurred as there are terraces of different sizes, reflected in the fact that the peak is now much broader than, e.g., in Fig. 4, its limits being the smaller and the larger terrace sizes. For the remainder, everything can be explained in terms of the picture we are providing. In view of all this, we can conclude that the smoothing process we are reporting and its interpretation are fully consistent with all the simulation data.

\section{B. Finite temperature}

In the previous subsection we were to identify the relevant features in the smoothing process at zero temperature. We now consider finite temperature effects. We propose that our simulations confirm the conclusion al-

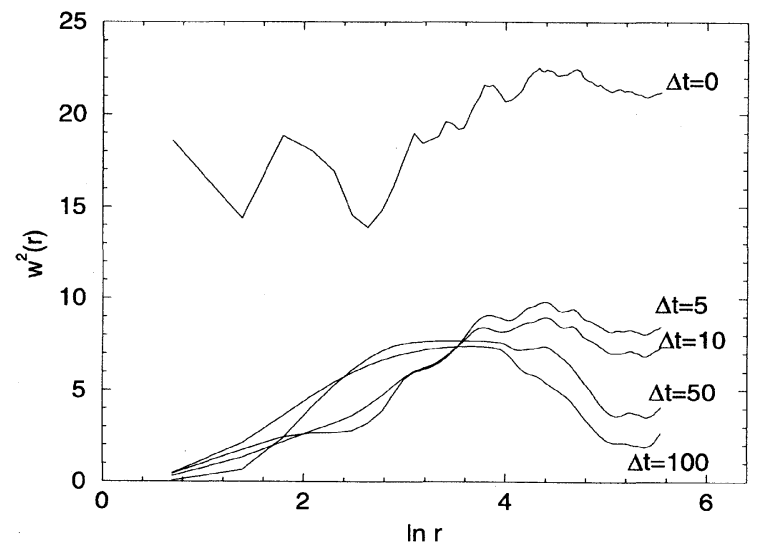

FIG. 15. Log-normal plot of $w^{2}(r)$ at the same times given in Fig. 13. ready obtained from the analysis of single terraces: For finite but low temperatures, everything occurs as in the $T=0$ case, whereas above a temperature around $T \sim 10$ in our units, thermal fluctuations mask the process and make it impossible to faithfully monitor the smoothing. We have verified this by following the evolution of the same initial data for both zero and finite temperatures. As an example, Fig. 16 summarizes the behavior of global quantities for the same initial data in Fig. 12, but now quenched to $T=5$. As is apparent from this figure, even if there is some thermal blurring, the process is basically the same as we discussed in the previous paragraphs. The only major difference is that now the terraces also show stretched exponential behavior. This is due to the fluctuations of the tops of the terraces, which now contribute to this value, while its contribution decreases with the size of the terraces. Therefore the evolution of this quantity is also a many scale process resulting in its functional shape. The rest of our indicators behave as explained, and terraces even nucleate around the same places (at least the not too small ones), and survive more or less for the same time [see Fig. 16(d) and compare to Fig. 12]. Hence our earlier conclusion that temperature, below a certain value, introduces nothing new but a blurring of the process and, to some extent, an extension of the lifetime of the surface.

\section{CONCLUSIONS}

In summary, we have used a 2D discrete sine-Gordon model to study how an initially rough surface becomes smoother when quenched below the roughening temperature. We approached this problem in the same spirit as Ref. 10. We began by analyzing the evolution of an artificially produced circular terrace at finite (but below $T_{R}$ ) temperature. We found that, in our model, the relationship between the terrace radius and the time it takes to disappear is $R \sim t^{2}$ at zero temperature, instead of the prediction in Ref. 10 of $R \sim t^{3}$, and closer to the results of Ref. 7 for sinusoidal grooves. If the temperature at which the surface is quenched is small but nonzero, this exponent is modified, its value being around 2.27 for $T=5$. However, finite temperature effects include a large dispersion between different numerical realizations, which calls for much more statistics that we can achieve with our current computational capabilities. We can only conclude that if $T \lesssim 0.1 T_{R}$, the exponent is $2.05 \pm 0.05$ (for our model). This suggests that the analytical treatment in Ref. 10 may not be complete, as indeed the author suggests. On the other hand, even if these predictions do not match our results for single terrace decay, we stress that our simulations of smoothing of a rough surface provide a qualitative picture that is very close to that of Ref. 10, and we can confirm the validity of the conjectures therein, particularly Fig. 4, which is the same as our Fig. 14 for the height-difference correlation function $C(r)$.

We identified the existence of three stages in the smoothing process: decay of the smallest fluctuations, reshaping of the largest terraces, and individual decay of each terrace. Therefore our results are consistent 

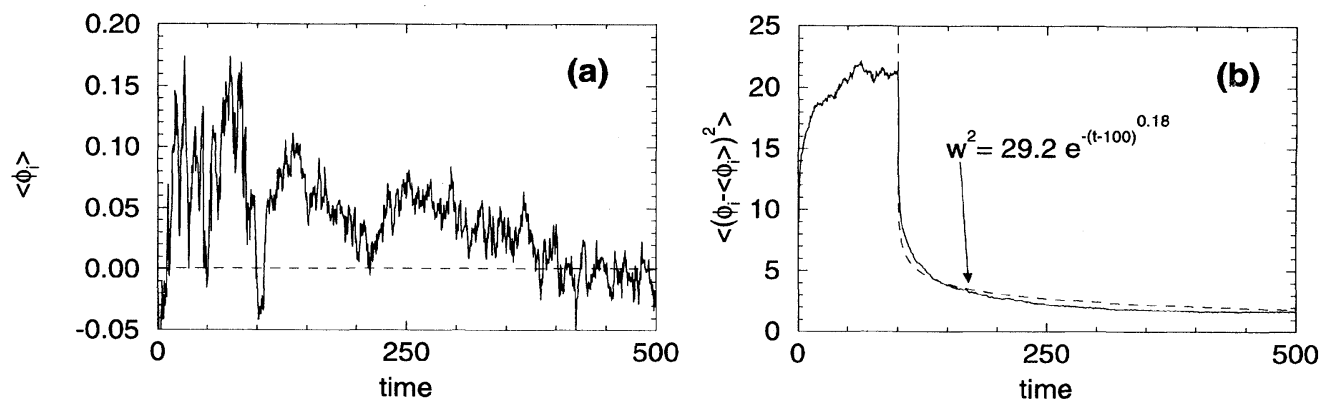

FIG. 16. Decay of a rough surface at $T=5$. The initial data are as in Fig. 12. Shown are the mean height (a), the mean roughness (b), the mean
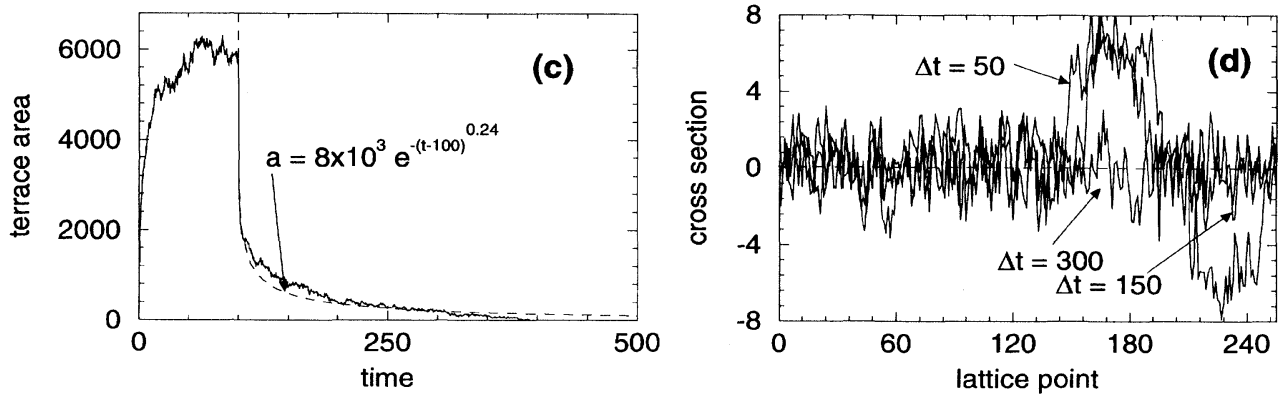
area of the terrace as given by the surface area above $2 \pi$ (c), and the evolution of a cross section (through column 15) as given by three snapshots taken at the indicated times (d).

with Villain's approach ${ }^{10}$ in that, after a transient, the smoothing process is understandable to a good approximation in terms of non-interacting terrace dynamics. Interestingly, we have also provided evidence that such a superposition of independent evolutions leads to glassylike dynamics, as it involves many different length scales (the larger the rough surface, the larger the number of scales involved). In connection with this point, we note that experiments designed to test these ideas are possible with currently available techniques: Thus roughness can be measured by means of scanning tunneling microscopy (STM), reflection high energy electron diffraction (RHEED), or other spectroscopic methods. ${ }^{2,19}$ It would be most interesting to address this question experimentally to verify whether smoothing is indeed a glassylike process.

We close with a few comments regarding open questions and extensions of our work. As an immediate unsolved issue, the problem of the exponent of the radiussurvival time relationship remains open; the predicted appearance of glassy dynamics in smoothing is now a question for experiments to verify. There are two other interesting extensions of this work, namely, the search for indicators (e.g., coarse-graining, image analysis) to study smoothing at higher temperatures (of the order of half the roughening temperature) and the analysis of smoothing in the presence of a driving force (e.g., chemical potential difference, for instance; see Ref. 5 for a discussion of this term), which would be relevant to thin film and other surface growth problems and their technologi- cal applications. We also note two factors that should be included in our model that can significantly modify our current conclusions. The first of these is disorder. The presence of foreign atoms or vacancies in the substrate is a crucial factor, as they can act as pinning centers for the shrinking terraces, halting their decay and leaving the surface in a stable, or metastable nonflat final condition. Such a rough shape can be desirable or undesirable depending on the application one has in mind, and therefore it may be interesting to fabricate disordered or very clean substrates, as well as to learn the consequences of any unavoidable amount of imperfections. The other factor relevant to actual growth processes is chemistry. It is evident that in very many film fabrication techniques chemical reactions play a crucial role in determining the final surface texture. This is absent in our model, which at most incorporates chemical effects through the driving force term. It would be very interesting to identify another "canonical" (in the same sense as our model) reaction-diffusion equation to couple to our sine-Gordon model accounting for chemical reactions. A more quantitative comparison of our predictions with many real processes would then be possible.

\section{ACKNOWLEDGMENTS}

Work at Los Alamos is performed under the auspices of the U.S. DOE. Work by A.S. was also supported by MEC (Spain)/Fulbright at Los Alamos and by CICyT (Spain) through project MAT95-0325 at Leganés. 
${ }^{1}$ Solids Far From Equilibrium: Growth, Morphology and Defects, edited by C. Godrèche (Cambridge University, New York, 1991).

${ }^{2}$ Mechanisms of Thin Film Evolution, edited by S. M. Yalisove, C. V. Thompson, and D. J. Eaglesham, MRS Symposia Proceedings No. 317 (Materials Research Society, Pittsburgh, 1994).

${ }^{3}$ W. K. Burton, N. Cabrera, and F. C. Frank, Philos. Trans. R. Soc. London Ser. A 243, 299 (1951).

${ }^{4}$ J. D. Weeks and G. H. Gilmer, Adv. Chem. Phys. 40, 157 (1979); H. van Beijeren and I. Nolden, in Structure and Dynamics of Surfaces, edited by W. Schommers and P. Von Blackenhagen, Topics in Current Physics Vol. 43 (Springer, Berlin, 1987).

${ }^{5}$ A. Sánchez, D. Cai, N. Grønbech-Jensen, A. R. Bishop, and Z. J. Wang, Phys. Rev. B 51, 14664 (1995).

${ }^{6}$ W. W. Mullins, J. Appl. Phys. 30, 77 (1959).

${ }^{7}$ G. Martin and B. Perraillon, Surf. Sci. 68, 57 (1977).

${ }^{8}$ F. Lançon and J. Villain, Phys. Rev. Lett. 64, 293 (1990).

${ }^{9}$ W. Selke and P. M. Duxbury, Z. Phys. B 94, 311 (1994);
Acta. Phys. Slovaca 44, 215 (1994).

${ }^{10}$ J. Villain, Europhys. Lett. 2, 531 (1986).

${ }^{11}$ F. Falo, A. R. Bishop, P. S. Lomdahl, and B. Horovitz, Phys. Rev. B 43, 8081 (1991).

${ }^{12}$ Z. J. Wang, P. S. Lomdahl, and A. R. Bishop (unpublished).

${ }^{13}$ S. T. Chui and J. D. Weeks, Phys. Rev. Lett. 40, 733 (1978).

${ }^{14}$ C. C. Hsu, Y. C. Lu, J. B. Xu, and I. H. Wilson, Appl. Phys. Lett. 64, 1959 (1994); C. C. Hsu, J. B. Xu, and I. H. Wilson, ibid. 64, 2105 (1994).

${ }^{15}$ About 2 CPU ms per lattice site per unit time on a SparcStation 2000 or a HP $9000 / 715 / 75$.

${ }^{16}$ H. S. Greenside and E. Helfand, Bell Syst. Tech. J. 60, 1927 (1981).

${ }^{17}$ N. Grønbech-Jensen, A. R. Bishop, F. Falo, and P. S. Lomdahl, Phys. Rev. B 45, 10139 (1992); 46, 11149 (1992).

${ }^{18}$ J. Feder, Fractals (Plenum Press, New York, 1988); K. J. Falconer, Fractal Geometry (Wiley, Chichester, 1990).

19 J. Villain, J. Phys. (France) I 1, 19 (1991). 


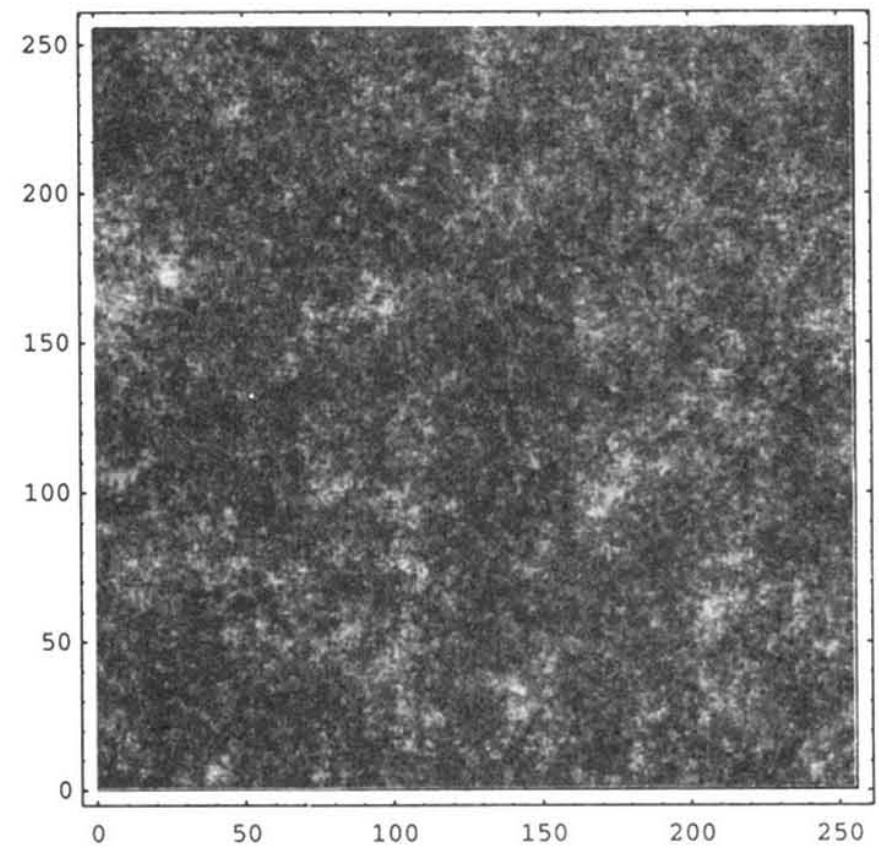

(a)

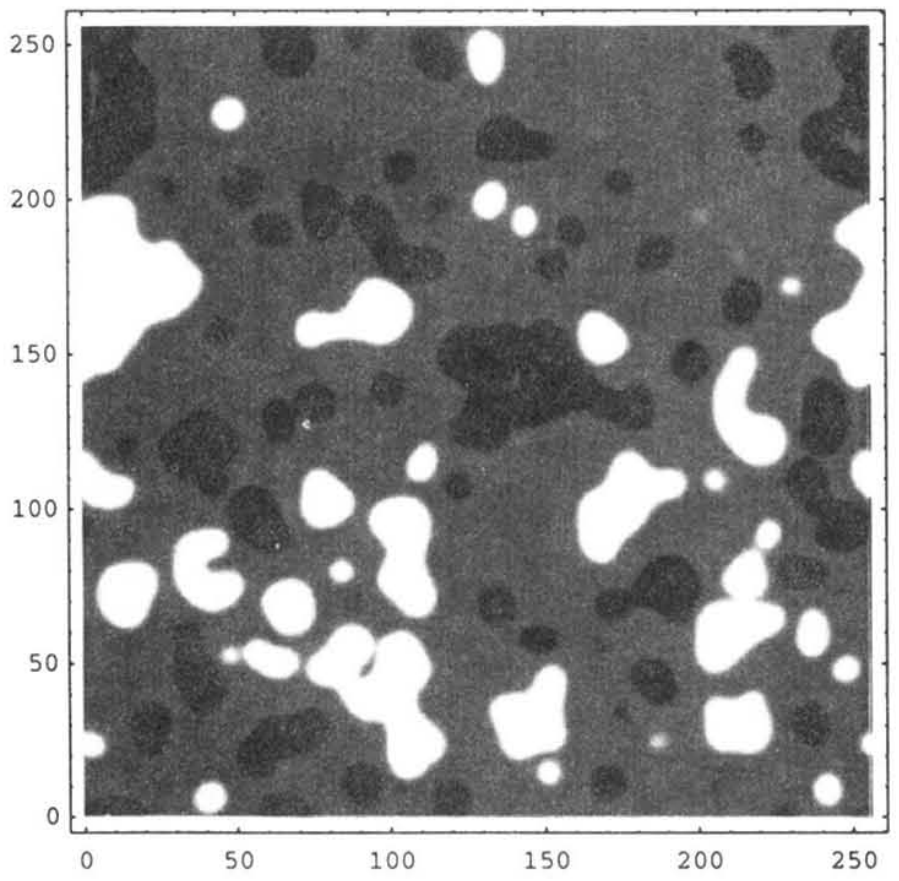

(c)

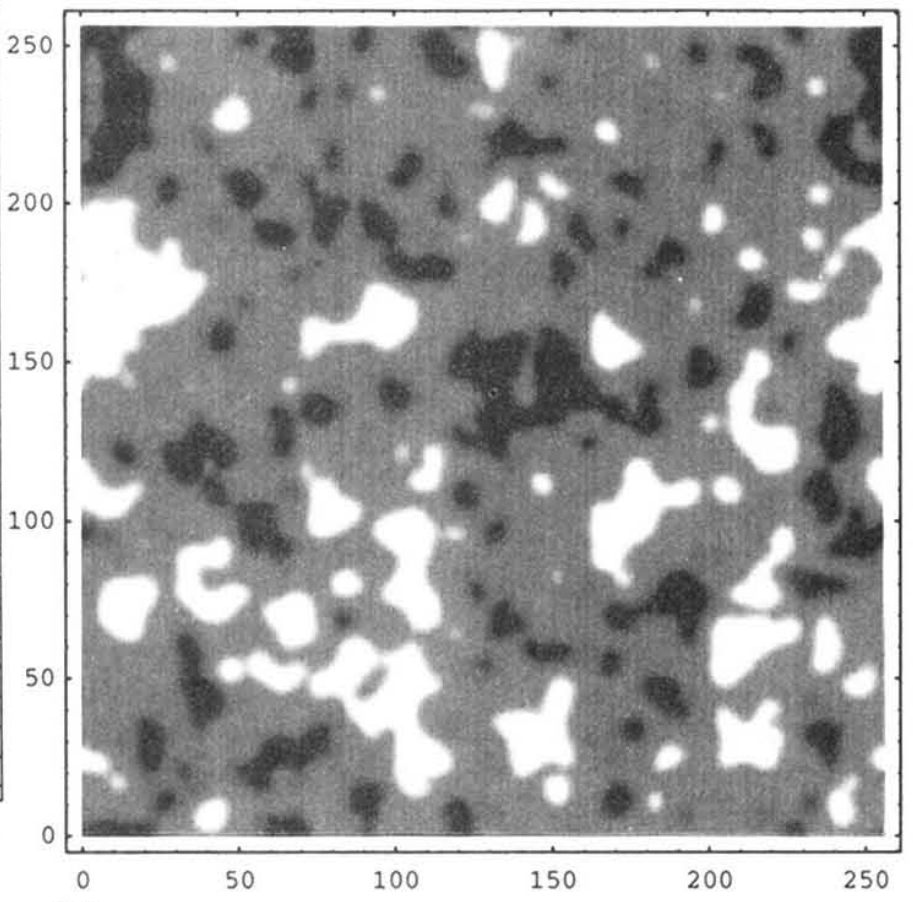

(b)

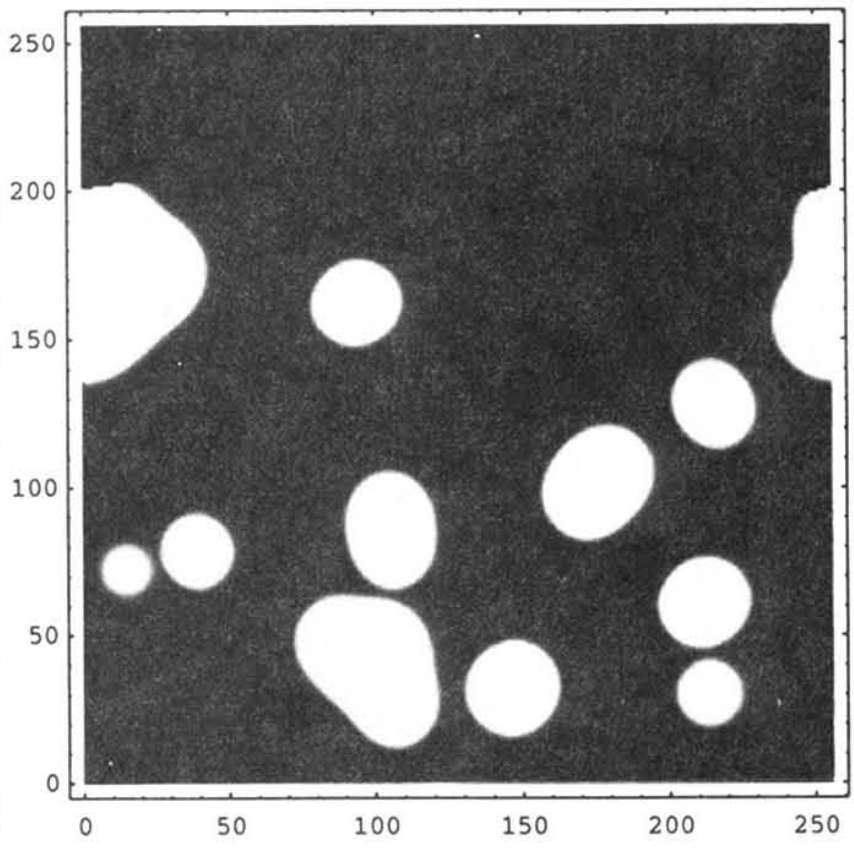

(d)

FIG. 13. Contour plots of the shape of the surface at times immediately before quenching (a), $t=5$ after the quench (b), $t=10$ after the quench (c), $t=50$ after the quench (d), and $t=100$ after the quench (e). The grayscale codes height such that lighter areas correspond to points with larger height. Axes simply indicate the coordinates of the lattice node, and periodic boundary conditions must be kept in mind. The evolution is the same as in Fig. 12. 


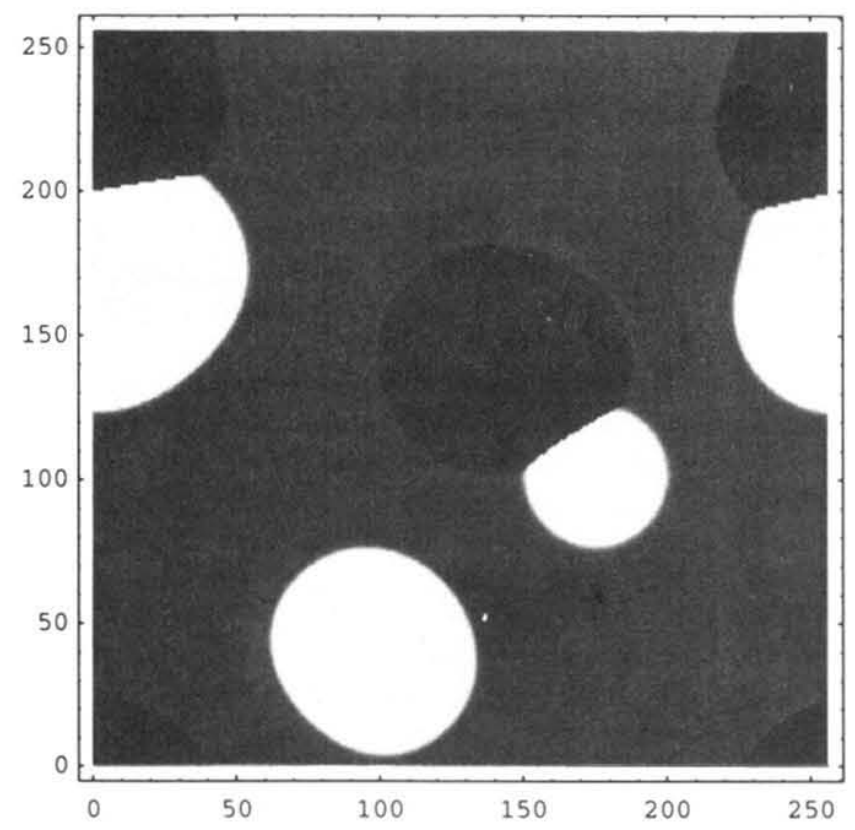

(e)

FIG. 13 (Continued). 